\title{
"Cerclaje", cierre o sutura del cuello incontinente ${ }^{\star}$
}

\author{
Dr. Rafael Peralta Cayón
}

Del Instituto Materno-Infantil - Bogotá

El tratamiento de la incontinencia cervical se basa en cerrar quirúrgicamente el cuello, como lo preconizan Shirodkar en la India, Lash y Lash en Estados Unidos, Palmer y Jácome en Francia, quienes hicieron la respectiva publicación en 1950, GreenArmytage en Inglaterra, Mc Donald en Australia, Mey en Alemania, Baden y Baden en Norte América, con éxitos del 50 al $85 \%$.

\section{OPERACION DE SHIRODKAR}

Fue la primera que se conoció al ser presentada al Congreso de Ginebra en 1954, y puede realizarse fuera de la gestación o durante esta, entre las semanas 16 a 19, con éxito hasta del $100 \%$, en cuanto a conservación del embarazo se refiere.

\section{TECNICA}

Anestesia general o local. Posición de Trendelembourg; incisión transversa de $3 \mathrm{cms}$. encima del labio anterior del cuello, al nivel del orificio interno, previa aplicación de valvas vaginales; disociación, rechazo de la vejiga, e incisión vertical de unos 2 a $3 \mathrm{cms}$. atrás, en el labio posterior; paso de una seda número $7 \mathrm{u}$ 8 de arriba hacia abajo por el lado izquierdo, para llevarla luego de abajo a arriba por el borde lateral derecho, y anudar adelan-

* El presente estudio fué presentado al IV Congreso Colombiano de Obstetricia y Ginecología. - Barranquilla, 1959. - En consecuencia, es la primera comunicación dada a conocer en Colombia sobre el tema. 
te y arriba donde se hizo la primera incisión. Luego reposo, sedantes, la sutura se seccionará en el momento del trabajo, o a la $31^{\text {a }}$ semana de embarazo.

\section{MATERIAL Y RESULTADOS}

Nueve casos operados por Shirodkar de la $12^{\mathrm{a}}$ a la $20^{\mathrm{a}}$ semanas, llegaron a término, 3 con Cesáreas, 6 por vía baja, y en 5 se observó que el conato de aborto, desapareció.

En base a la intervención anterior, se han practicado otras, con algunas modificaciones. V. B. Green-Armytage y Mc Donald, publicaron 70 casos entre las 20 y 24 semanas, con 33 niños vivos; en 16 se prolongó el embarazo por 4 semanas solamente, pero hubo supervivencia fetal, para concluír que hay éxito si la sutura alcanza más de 5 semanas, y que las contraindicaciones son, toxemia, hidramnios, metrorragia y ruptura prematura de la bolsa.

A. F. Lash, quien primitivamente propuso intervenir fuera del embarazo, relata 29 gestantes operadas, de las cuales 23 llegaron al término o casi al término, 4 de ellas requirieron segunda o tercera intervención, y al tiempo hubo dos abortos. En total los partos fueron 27. por vía baja 16 y con Cesárea 11, y 21 hijos vivos. Si a éstos se agregan observaciones de pacientes operadas fuera de la preñez, se completan 44, con un balance de $61 \%$ de éxito global, que suben a $93 \%$, si únicamente se consideran las 29 historias que alcanzaron al final del embarazo.

Robert H. Baster y James A. Dusbabek, Humbert L. Riva, John Parks, relatan 22 experiencias, (19 de tres hospitales donde habían ocurrido 35.000 partos), que tenían 91 embarazos previos con solo 10 hijos vivos, que luego alcanzaron 14 partos, 10 por vía alta y 5 por vía baja, todos con fetos vivos, faltando por contabilizar 2 casos que aún no habían tenido los respectivos partos. Aconsejan intervenir de la $14^{\mathrm{a}}$ a $18^{\mathrm{a}}$ semanas; hacen las incisiones transversalmente tomando los labios del cuello, con pinzas de corazón u ovaladas, y después de esos 32 casos Baster ha preferido utilizar material no absorbible, Mersiline (Dacron), en 14 observaciones, advirtiendo que también se ha usado material de talco, o alambre tantalium, y Page, quien opera fuera del 
embarazo, emplea catgut, más talco, porque éste excita los histiocitos y da un tejido escleroso más espeso, más fuerte, para cerrar el cuello.

Charles L. Easterday y Duncan E. Reid, suturaron 18 casos con alambre maleable número 0 , en gestantes de diferentes edades.

\section{CUADRO No 1}

Semana de Embarazo

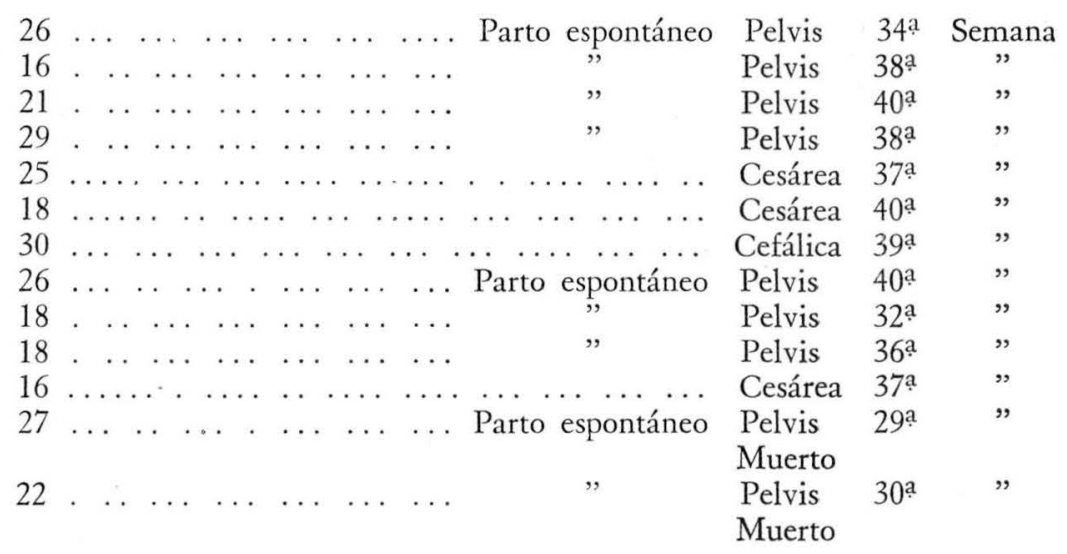

NOTA: De las observaciones que terminaron con partos y fetos vivos, tenían como máximo 1 solo hijo vivo, y antecedentes de varios abortos o prematuros. Con el procedimiento quirúrgico aumentaron los hijos vivos y salvados, ya que apenas se perdieron 3 , por prematurez.

Por último, Paimer y colaboradores, frente a la incontinencia de cuello, establecen como norma terapéutica, suministrar tiamina a dosis elevada, y Progesterona, que suben el tono ístmico, cuando no hay causa traumática, pero si ésta se hace ostensible, preconizan la cirugía en la siguiente forma:

a) Traquelorrafia amplia, contra el desgarro, mediante disección, avivamiento, en dos planos, y puntos separados con catgut número 2 sobre bujía de Hegar 4, suturando bien vagina y región supravaginal del cuello. 
b) Istmorrafia, para la abertura exclusiva del istmo, con avivamiento-sutura, o con pliegues cosidos en el istmo-cuello, previa colpotomía anterior en $\mathrm{T}$ invertida, y disección, rechazo vesical, también sobre bujía de Hegar, sea fuera del embarazo o en seguida del aborto, aprovechando la blandura de los tejidos, o al segundo mes de gestación; todo con 3 éxitos publicados.

c) Cerclaje cérvico-ístmico de Shirodkar, aun existiendo prolapso de las membranas, pero dejando el nudo hacia atrás, a fin de que éste no irrite la vejiga o uretra, y empleando bandas de seda de $3 \mathrm{cms}$. de ancho, o de fascia lata, más Cesárea si no se quiere repetir después la operación.

\section{Nuestra Experiencia en Sutura del Cuello Uterino Gestante Incontinente}

Presentamos a continuación dos casos intervenidos durante el embarazo, entre diez o más que hemos registrado en total.

\section{HISTORIA CLINICA No 1}

C. A. C. de B. Edad: $40 \frac{1}{2}$ años. Menarquia: $13 \frac{1}{2}$ años, 3, 4/29. Dismenorreas, metrorragias. Tres partos a término. Nueve abortos, la mayoría de $3 \frac{1}{2}$ a 5 meses, espontáneos, con prolapso de membranas, fetos vivos y extracciones manuales de la placenta.

Diagnóstico inicial en Junio 17/59: multigestante XIII de 2 meses. Aborto habitual por incontinencia de cuello uterino.

Evolución: U. R.; en Marzo o Abril 17/59. Desde un principio por si existía fibrosis endometrial, se sometió a tratamiento con derivados corticosteroides, (Medrol). Desde un comienzo se propuso el Cerclaje cervical, que fue aplazando la paciente "por sentirse bien", y por no tener la aceptación del esposo. Al final de Agosto hubo contracciones uterinas dolorosas, flujo sanguinolento, y hasta Septiembre 16 fue cuando se operó.

Bajo anestesia general se verificó el Cerclaje del cuello uterino gestante, modelo SHIRODKAR, con aguja DOYEN, que resultó corta y motivó sección lateral derecha en el tejido cervical, anudando con nylon 3 doble hacia atrás, y habiendo quedado cerrado el cuello en forma que pareció satisfactoria. Enseguida antibióticos parenterales, sulfatiazol local, calmantes a base de Suposedol, y 3 días en la Clínica en buenas condiciones. Salió a la casa. pero desde los 5 días de la intervención hubo metrorragia dolorosa, tratada con los medicamentos habituales hasta el 29 de Septiembre, 13 días después de la operación, en que se presentó un parto con feto inmaduro, de 680 grs., vivo, 
masculino, que vivió una hora. Una hora y media después del parto hubo que hacer alumbramiento artificial, previa transfusión de 500 c.c. de sangre. Al revisar, se observó que el nylon trozó parte del cuello y fue a un lado de éste, y después se extirparon el fragmento de tejido cervical sobrante y el hilo de sutura. La biopsia, dio endocervicitis aguda, restos de embarazo con reacción inflamatoria y vasos sanguíneos esclerosados; no hay cambios de colagenosis; decidua con inflamación, degeneración, necrosis y hialinización; vellosidades coriales pequeñas.

Causa del fracaso: Operación algo tardía?, o quedó el Cerclaje muy bajo infra ístmico? Aunque el diagnóstico de Incontinencia es indudable, influirá en el aborto habitual la edad del marido, sesenta años confesados, sin haber atendido la orden de espermograma?

\section{HISTORIA CLINICA No 2}

A. B. V: de D. Historia del Instituto Materno Infantil No 65575. Edad: 25 años. Regla: 13 años, 5/29. Amenorreas y dismenorreas.

Diagnóstico inicial: Aborto habitual primario por INCONTINENCIA DE CUELLO.

Antecedentes obstétricos: Ningún hijo vivo. Cuatro abortos, los dos primeros de 4 meses, y los dos últimos de $4 \frac{1}{2}$ meses, el tercero, gemelar; ha habido en ellos prolapso de las membranas previamente, y los fetos han nacido vivos. Antes del cuarto embarazo, en el curso de una laparotomía se practicó resección ovárica cuneiforme bilateral, y durante toda esa gestación, se hizo terapia corticosteroide, porque se consideró la posibilidad de fibrosis endometrial. Después del cuarto aborto se comprobó, en definitiva, la existencia de cuello uterino incontinente.

Evolución actual: U. R. Junio 13/59. Quinto embarazo normal. El 13 de Octubre del 59, bajo anestesia general se practica Cerclaje, con técnica similar a la del caso anterior.

RESULTADO: Se hicieron controles periódicos, cada 10 y 15 días después de la operación. Al revisar con espéculo, nada especial se apreció; la auscultación fetal siempre fue positiva y no hubo amenaza de interrupción del embarazo. Los tratamientos que se hicieron, fueron los rutinarios de la preñaz normal.

\section{EPILOGO EN 1960:}

El 6 de Marzo, con embarazo de 81/2 a 9 meses, a las 5 de la mañana tuvo ruptura prematura de la bolsa de las aguas. A las 6 y 30 se iniciaron las contracciones uterinas, con ligera metrorragia. A las 11 la dilatación cervical era de $3 \mathrm{cms}$., con bolsa rota y contracciones irregulares. Bajo anestesia general se quitó el nylon y se hizo Cesárea segmentaria transperitoneal, feto masculino de $2.900 \mathrm{grs}$. en asfixia, se reanimó a los 9 minutos con los trata- 
mientos habituales. Una circular del cordón. Permanencia en la Clínica durante 6 días. de donde salieron en magníficas condiciones tanto la madre como el hijo, los cuales se han controlado después y están muy bien.

\section{COMENTARIO}

Es un caso de éxito absoluto, tal vez el primero que se publica en Colombia, ya que al revisar la literatura nacional no conocemos otro igual, operado durante la gestación, y que luego esta haya llegado a su final. con un balance materno-fetal satisfactorio.

El diagnóstico de la incontinencia cervical es irrefutable; se operó a los 31/2 meses de embarazo, y con ello se logró por primera vez un hijo casi a término, que vive, cuando los cuatro embarazos anteriores se habían frustrado con abortos habituales.

Se dudó si el parto podía conducirse por vía vaginal o con Cesárea. Pensamos en la posibilidad, de que el proceso largo del parto por vía baja, pudiera aumentar más la incontinencia que corregimos, o pudimos mejorar con la sutura cérvico-ístmica. A posteriori, con la circular del cordón, la muerte aparente del niño, quedamos satisfechos de haber preferido la Cesárea. Por último, en futuros embarazos habrá que repetir el Cerclaje? Sería probable, pero es mejor esperar y observar en concreto el estado en que haya quedado el cuello, para definir esto. Eso sí, en otro parto, después de soltar el hilo, podría realizarse por vía natural, y no con Cesárea.

\section{COMENTARIO (Marzo 13-61)}

La paciente de la primera observación ha vuelto a quedar embarazada. En cuanto a la segunda, en Nov. 8-60, se diagnosticó nueva gestación de 21/2 meses, U. R. Agosto 13-60. Se ha controlado en Enero 11, Febrero 20 y Marzo 13-61, en esta última fecha embarazo normal de $6 \frac{1}{2}$ a 7 meses, normal. Desde un principio se revisó cuello, y pareció bien. Ex-profeso, no se quiso repetir "cerclaje", de acuerdo con la tesis expuesta. Parece que podemos estar en lo cierto, y es preferible la Cesárea, sobre todo en aborto habitual primario, si se quiere evitar nuevo "cerclaje", cuya técnica, por lo sangrante, no es tan fácil como se describe usualmente. 
Vol. XII

\section{BIBLIOGRAFIA}

BADEN W. F. BADEN E. E. "Cervical incompetence: Repair during pregnancy”. Am. J. Obst. y Gyn. Vol. 74, N 2, Aug.-57, p. 241.

BARTER H. R., DUSBABEK J. A., RIVA H. L., PARKS J. "Surgical closure of the incompetent cervix during pregnancy". Am. J. Obs. y Gynec. Vol. 75, No 3, March-58, p. 511.

DANFORT DAVID N. "Symposium on spontaneous abortion". March. 1959.

EASTERDAY CHARLES. "The incompetent cervix in repetive abortion and premature labor". The New England. Journal of Med. Vol. 1260, No 14, Apr. 2-59, p. 687.

Encyclopedie Medico-Chirurgicale. Obstetrique. "Avirtement" par beance de l'isthme", 1er. d, 5-1958 C. 5075 B 10, p. 4 c, 5, 9 f, 10.

JAVERT CARL J. "Spontaneous and habitual abortion". The Blackiston Division Mcgrew-Hill Book Company, Inc. N. York, Toronto, London 1957.

Year Book Obstetrics Gynecology. Greenhill, 58-59, "Suture of cervix for in evitable miscarriage". p. 52. Traction TEST for demostrating incompetence os of cervix, p. 53. 